\title{
Two New Sliding DTFT Algorithms for Phase Difference Measurement Based on a New Kind of Windows
}

\author{
Yaqing Tu${ }^{1}$, Ting'ao Shen ${ }^{1,2}$, Haitao Zhang ${ }^{1}$, Ming $\mathrm{Li}^{1}$ \\ ${ }^{1}$ Department of Information Engineering, Logistical Engineering University, Chongqing 401311, China \\ ${ }^{2}$ Corresponding author: peoplesta@sina.com
}

For the ultra-low frequency signals or adjacent Nyquist frequency signals, which exist in the vibration engineering domain, the traditional DTFT-based algorithm shows serious bias for phase difference measurement. It is indicated that the spectrum leakage and negative frequency contribution are the essential causes of the bias. In order to improve the phase difference measurement accuracy of the DTFT-based algorithm, two new sliding DTFT algorithms for phase difference measurement based on a new kind of windows are proposed, respectively. Firstly, the new kind of windows developed by convolving conventional rectangular windows is introduced, which obtains a stronger inhibition of spectrum leakage. Then, with negative frequency contribution considered, two new formulas for phase difference calculation under the new kind of windows are derived in detail. Finally, the idea of sliding recursive is proposed to decrease the computational load. The proposed algorithms are easy to be realized and have a higher accuracy than the traditional DTFT-based algorithm. Simulations and engineering applications verified the feasibility and effectiveness of the proposed algorithms.

Keywords: Phase difference, discrete time Fourier transform, cosine window, spectral leakage, negative frequency.

\section{INTRODUCTION}

$\mathrm{T}^{\mathrm{s}}$ HE PROBLEMS of estimating the phase difference between signals received at two separated sensors are considered in many areas such as fault diagnosis, direction finding, and source localization. Many methods have been proposed for phase difference measurement in the past three decades [1]-[6]. Generalized Cross Correlator (GCC) [7]-[9] is a conventional approach to estimate the phase difference by locating the cross correlation peak of the filtered version of two received signals, and it has been proven that optimum performance can be attained when the signals and noises are Gaussian distributed. However, the method requires a prior statistics of the received signals and it fails to work when the noises are impulsive or spatially correlated.

When the source signals are deterministic, a Discrete-time Fourier transform (DTFT)-based algorithm is proposed for single complex sinusoids [10], while a Quadrature Delay Estimator algorithm is developed for real-valued sinusoids by utilizing the in-phase and quadrature-phase components of one of the received signals [11]. Using the idea of [11], two modified methods have been developed for real sinusoidal signals [12]. The first method is referred to as UQDE, which removes the bias of QDE by utilizing all the in-phase and quadrature-phase components of the received signals. The second method is referred to as modified DTFT algorithm, which calculates the phase difference of DTFTs of two complex sinusoids derived from the real signals. The key idea of the modified DTFT algorithm is to transform the real tone to a complex one with the known frequency information, and this is the difference between the modified DTFT algorithm and the typical DTFT-based algorithm. However, all the algorithms are difficult to achieve in practice if the frequency is unknown.

Among the phase difference measurement methods for real signals with unknown frequency, the Discrete-time Fourier transform-based algorithm is used as the typical one.
The DTFT-based algorithm calculates the phase difference of DTFTs of two real sinusoids at the estimated signal frequency. However, the algorithm neglects the contribution of negative frequency for real-valued sinusoids. When the signal frequency is quite low or close to the Nyquist frequency, the algorithm brings about significant bias or even becomes ineffective. The same thing occurs when the available sampled data for DTFT calculation are not enough.

To remove the bias and improve the accuracy of the DTFT-based algorithm, two new sliding DTFT algorithms are proposed in this paper. In Section 2, the proposed algorithms and the process of their application are described in detail, which introduce the new kind of windows, the windowed DTFT algorithms with negative frequency contribution and the idea of sliding recursive, respectively. In Section 3, the proposed algorithms are validated by simulations and experiments. Finally, Section 4 presents the conclusion.

\section{THE PRINCIPLE OF THE PROPOSED ALGORITHMS AND THEIR APPLICATION PROCESS}

\section{A. A new kind of windows and its characteristic.}

A new kind of windows, called convolved-windows, which is developed by convolving conventional rectangular window, was introduced in [13]. This kind of windows has the advantages of simple structure, lower attenuation and good inhibition of spectral leakage. The construction of the convolved-windows can be expressed as follows.

Consider the rectangular window time domain and frequency domain expressions as

$$
\begin{gathered}
w_{R}(n)=1, \quad 0 \leq n \leq M-1 \\
W_{R}\left(e^{j \omega}\right)=\frac{\sin (M \omega / 2)}{\sin (\omega / 2)} e^{-j \frac{M-1}{2} \omega}
\end{gathered}
$$


where $M$ is the length of rectangular window, and $\omega$ is the digital signal frequency. A new $2 M-1$ data sequence can be obtained by convolving 2 rectangular windows. If 1 zero is added in the front or back of the new sequence, a new $2 M$ data sequence can be obtained, called 2-order rectangular self-convolution window (RSCW). Add 1 zero in the front of the $2 M-1$ data sequence and consider $N=2 M$, the time domain and frequency domain expressions of 2-order RSCW can be expressed as

$$
\begin{gathered}
w_{R^{2}}(n)= \begin{cases}n & 0 \leq n \leq N / 2-1 \\
N-n & N / 2 \leq n \leq N-1\end{cases} \\
W_{R^{2}}\left(e^{j \omega}\right)=\frac{\sin ^{2}(N \omega / 4)}{\sin ^{2}(\omega / 2)} e^{-j \frac{N}{2} \omega}
\end{gathered}
$$

Similarly, $4 M-3$ data sequence can be obtained by convolving 4 rectangular windows. A new 4-order RSCW can be developed by adding 2 zero in the front of the new sequence and 1 zero at the back of the new sequence. Consider $N=4 M$, the time domain and frequency domain expressions can be expressed as

$$
w_{R^{4}}(n)=\left\{\begin{array}{l}
\frac{1}{6} n^{3}-\frac{1}{6} n \quad 0 \leq n \leq \frac{N}{4}-1 \\
-\frac{1}{2} n^{3}+\frac{N}{2} n^{2}+\left(\frac{1}{2}-\frac{N^{2}}{8}\right) n+\left(\frac{N^{3}}{96}-\frac{N}{6}\right) \quad \frac{N}{4} \leq n \leq \frac{N}{2}-1 \\
\frac{1}{2} n^{3}-N n^{2}+\left(\frac{5 N^{2}}{8}-\frac{1}{2}\right) n+\left(\frac{N}{3}-\frac{N^{3}}{96}\right) \quad \frac{N}{2} \leq n \leq \frac{3 N}{4}-1 \\
-\frac{1}{6} n^{3}+\frac{N}{2} n^{2}+\left(\frac{1}{6}-\frac{N^{2}}{2}\right) n+\left(\frac{N^{3}}{6}-\frac{N}{6}\right) \quad \frac{3 N}{4} \leq n \leq N-1
\end{array}\right.
$$

$$
W_{R^{4}}\left(e^{j \omega}\right)=\frac{\sin ^{4}(N \omega / 8)}{\sin ^{4}(\omega / 2)} e^{-j \frac{N}{2} \omega}
$$

According to the construction of the RSCW, the $m$-order RSCW can be developed in a similar way. At first, a new data sequence can be obtained by convolving $m$ rectangular windows, and then, if $m$ is even, the $m$-order RSCW can be developed by adding $m / 2$ zero in the front of the new sequence and $(m-2) / 2$ zero at the back of the new sequence, while $m$ is odd, the $m$-order RSCW can be developed by adding $(m-1) / 2$ zero in the front of the new sequence and $(m-1) / 2$ zero at the back of the new sequence, and then, the time domain and frequency domain expressions of $m$-order RSCW $(N=m \cdot M)$ can be expressed as

$$
W_{R^{m}}\left(e^{j \omega}\right)=\left\{\begin{array}{ll}
\frac{\sin ^{m}(N \omega / 2 m)}{\sin ^{m}(\omega / 2)} e^{-j \frac{N}{2} \omega} & \text { if } m=2 k \\
\frac{\sin ^{m}(N \omega / 2 m)}{\sin ^{m}(\omega / 2)} e^{-j \frac{N-1}{2} \omega} & \text { if } m=2 k+1
\end{array} \quad k \in Z^{+}\right.
$$

It is obviously from formula (7) that the width of the main lobe becomes larger with the increase of $m$, accordingly, the order of RSCW is usually less than 4. In addition, in the zero points of its amplitude-frequency characteristic, the value of $m-1$ order derivatives is zero. As a result of this character, the interferences between the harmonics due to spectrum leakage can be reduced furthest by applying the RSCW. Consequently, the precision of harmonics estimation can be boosted.

\section{B. The windowed DTFT algorithms.}

1) The measurement principle of DTFT algorithm

Consider two real sinusoids with the same frequency

$$
\begin{aligned}
& x_{1}(n)=A_{1} \cos \left(\omega \cdot n+\theta_{1}\right) \\
& x_{2}(n)=A_{2} \cos \left(\omega \cdot n+\theta_{2}\right)
\end{aligned}, \quad n=0,1, \cdots, N-1
$$

where $A_{1}$ and $A_{2}$ are amplitudes, $\theta_{1}$ and $\theta_{2}$ are initial phases. Mark $\hat{\omega}$ as the estimated value of $\omega$, the DTFT of $x_{1,2}(n)$ at $\hat{\omega}$ can be computed as [14]

$$
\begin{aligned}
X_{(1,2) N}(\hat{\omega}) & =\sum_{n=0}^{N-1} A_{1,2} \cos \left(\omega \cdot n+\theta_{1,2}\right) \cdot e^{-j \hat{\omega} n} \\
& =\sum_{n=0}^{N-1} \frac{A_{1,2}}{2}\left[e^{j\left(\omega \cdot n+\theta_{1,2}\right)}+e^{-j\left(\omega \cdot n+\theta_{1,2}\right)}\right] \cdot e^{-j \hat{\omega} n}
\end{aligned}
$$

According to Euler's formula, a real sinusoid can be formulated as the sum of two exponential signals with positive and negative frequencies, respectively. If the negative frequencies in (9) are neglected, then

$$
\begin{aligned}
X_{(1,2) N}(\hat{\omega}) & =\sum_{n=0}^{N-1} \frac{A_{1,2}}{2} e^{j\left(\omega \cdot n+\theta_{1,2}\right)} \cdot e^{-j \hat{\omega} n} \\
& = \begin{cases}\frac{A_{1,2}}{2} \frac{\sin \left(\frac{N(\hat{\omega}-\omega)}{2}\right)}{\sin \left(\frac{\hat{\omega}-\omega}{2}\right)} \cdot e^{j\left[\theta_{1,2}+\frac{(\hat{\omega}-\omega) N}{2}-\frac{\hat{\omega}-\omega}{2}\right]}, \text { if } \hat{\omega} \neq \omega \\
\frac{A_{1,2}}{2} \cdot N \cdot e^{j \theta_{1,2}}, & \text { if } \hat{\omega}=\omega\end{cases}
\end{aligned}
$$

The phase of $X_{1 N}(\hat{\omega})$ and $X_{2 N}(\hat{\omega})$ is denoted by $\varphi_{1}$ and $\varphi_{2}$, respectively. If the phase difference is defined as $\Delta \theta=\theta_{2}-\theta_{1}$, it can be calculated as 


$$
\Delta \theta=\varphi_{2}-\varphi_{1}
$$

This is the typical DTFT-based algorithm. It is obvious that the phase difference of two signals approximates the subtraction of two DTFT phases at the estimated signal frequency. If the negative frequency is neglected in (10), it is no longer the DTFT of the real sinusoidal signal but is that of the complex one. In other words, the contribution of negative frequency components in the spectrum is neglected in typical DTFT-based algorithm. When the signal frequency is quite low or close to the Nyquist frequency, the negative frequency interference in the spectrum becomes remarkable, which will bring about significant bias in phase difference measurement. The same thing will occur if a small number of sampled data are taken in DTFT calculation.

Thus, two new DTFT algorithms with negative frequency contribution considered, which aim to remove the bias of the DTFT-based algorithm, are presented. Two new formulas for phase difference measurement adopting 2-order RSCW and 4-order RSCW are derived, respectively.

2) The new DTFT algorithm based on 2-order RSCW

Assuming the length of 2-order RSCW is $N$, multiply the 2-order RSCW with signals $x_{1,2}(n)$, and the DTFT of the signals can be expressed as

$$
\begin{aligned}
& X_{R^{2},(1,2) N}(\hat{\omega})=\sum_{n=0}^{N-1} A_{1,2} \cos \left(\omega \cdot n+\theta_{1,2}\right) \cdot w_{R^{2}}(n) \cdot e^{-j \hat{\omega} n} \\
& =\sum_{n=0}^{N-1} \frac{A_{1,2}}{2}\left[e^{j\left(\omega \cdot n+\theta_{1,2}\right)}+e^{-j\left(\omega \cdot n+\theta_{1,2}\right)}\right] \cdot w_{R^{2}}(n) \cdot e^{-j \hat{\omega} n} \\
& =\frac{A_{1,2}}{2}\left[e^{j \theta_{1,2}} W_{R^{2}}(\hat{\omega}-\omega)+e^{-j \theta_{1,2}} W_{R^{2}}(\hat{\omega}+\omega)\right] \\
& =\frac{A_{1,2}}{2} \cdot \frac{\sin ^{2}\left(\frac{N(\hat{\omega}-\omega)}{4}\right)}{\sin ^{2}\left(\frac{\hat{\omega}-\omega}{2}\right)} \cdot e^{j\left[\theta_{1,2}-\frac{N(\hat{\omega}-\omega)}{2}\right]} \\
& +\frac{A_{1,2}}{2} \cdot \frac{\sin ^{2}\left(\frac{N(\hat{\omega}+\omega)}{4}\right)}{\sin ^{2}\left(\frac{\hat{\omega}+\omega}{2}\right)} \cdot e^{-j\left[\theta_{1,2}+\frac{N(\hat{\omega}+\omega)}{2}\right]}
\end{aligned}
$$

As is generally known, multiplying in time domain is in correspondence with convolving in frequency domain. So the equation 12 can be regarded as

$$
X_{R^{2},(1,2) N}(\hat{\omega})=X_{(1,2) N}(\hat{\omega}) * W_{R^{2}}(\hat{\omega})
$$

Assuming that $\hat{\omega} \neq \omega$, (13) can be derived from (12).

$$
\tan \varphi_{R^{2},(1,2) N}=\frac{\operatorname{Im}\left[X_{R^{2},(1,2) N}(\hat{\omega})\right]}{\operatorname{Re}\left[X_{R^{2},(1,2) N}(\hat{\omega})\right]}=\frac{c_{1} \tan \theta_{R^{2},(1,2) N}-c_{2}}{c_{3} \tan \theta_{R^{2},(1,2) N}+c_{4}}
$$

and then,

$$
\tan \theta_{R^{2},(1,2) N}=\frac{c_{4} \tan \varphi_{R^{2},(1,2) N}+c_{2}}{c_{1}-c_{3} \tan \varphi_{R^{2},(1,2) N}}
$$

where $\varphi_{R^{2},(1,2) N}$ denotes the phase of $X_{R^{2},(1,2) N}(\hat{\omega})$.

$c_{1}=\sin ^{2} a_{1} \sin ^{2} a_{4} \cos 2 a_{1}-\sin ^{2} a_{2} \sin ^{2} a_{3} \cos 2 a_{3}$

$c_{2}=\sin ^{2} a_{1} \sin ^{2} a_{4} \sin 2 a_{1}+\sin ^{2} a_{2} \sin ^{2} a_{3} \sin 2 a_{3}$

$c_{3}=\sin ^{2} a_{1} \sin ^{2} a_{4} \sin 2 a_{1}-\sin ^{2} a_{2} \sin ^{2} a_{3} \sin 2 a_{3}$

$c_{4}=\sin ^{2} a_{1} \sin ^{2} a_{4} \cos 2 a_{1}+\sin ^{2} a_{2} \sin ^{2} a_{3} \cos 2 a_{3}$

$a_{1}=N(\hat{\omega}-\omega) / 4, a_{2}=(\hat{\omega}-\omega) / 2$,

$a_{3}=N(\hat{\omega}+\omega) / 4, a_{4}=(\hat{\omega}+\omega) / 2$

Making use of the formula

$\tan \left(\theta_{R^{2}, 2 N}-\theta_{R^{2}, 1 N}\right)=\frac{\tan \theta_{R^{2}, 2 N}-\tan \theta_{R^{2}, 1 N}}{1+\tan \theta_{R^{2}, 2 N} \tan \theta_{R^{2}, 1 N}}$, the phase difference

can be deduced as:

$\Delta \theta=\arctan \left[\frac{\left(c_{1} c_{4}+c_{2} c_{3}\right)\left(\tan \varphi_{R^{2}, 2 N}-\tan \varphi_{R^{2}, I N}\right)}{\left(c_{1}^{2}+c_{2}^{2}\right)+\left(c_{2} c_{4}-c_{1} c_{3}\right)\left(\tan \varphi_{R^{2}, 1 N}+\tan \varphi_{R^{2}, 2 N}\right)+\left(c_{3}^{2}+c_{4}^{2}\right) \tan \varphi_{R^{2}, 1 N} \tan \varphi_{R^{2}, 2 N}}\right]$

When the signal-to-noise ratio (SNR) of signals is not very low, the signal frequency estimated by adaptive notch filter or discrete spectrum correction is generally quite close to the true value, i.e., $\hat{\omega} \approx \omega$, then, $\sin a_{1} / \sin a_{2} \approx N / 2$ can be deduced. And then, equation 15 can approximately be expressed as follows.

$$
\Delta \theta=\arctan \left[\frac{m_{1}\left(\tan \varphi_{R^{2}, 2 N}-\tan \varphi_{R^{2}, 1 N}\right)}{m_{2}+m_{3}\left(\tan \varphi_{R^{2}, 1 N}+\tan \varphi_{R^{2}, 2 N}\right)+m_{4} \tan \varphi_{R^{2}, 1 N} \tan \varphi_{R^{2}, 2 N}}\right]
$$

where

$m_{1}=(N \sin \hat{\omega})^{4} / 16-\sin ^{4} \beta$;

$m_{2}=(N \sin \hat{\omega})^{4} / 16+\sin ^{4} \beta-\left[(N \sin \hat{\omega} \sin \beta)^{2} \cos 2 \beta\right] / 2$;

$m_{3}=\left[(N \sin \hat{\omega} \sin \beta)^{2} \sin 2 \beta\right] / 2$;

$m_{4}=(N \sin \hat{\omega})^{4} / 16+\sin ^{4} \beta+\left[(N \sin \hat{\omega} \sin \beta)^{2} \cos 2 \beta\right] / 2$;

$\beta=N \hat{\omega} / 2$

Equation 16 is the new DTFT algorithm based on 2-order RSCW for phase difference calculation. When $\hat{\omega}=\omega$, the formula for phase difference calculation is just the same as (16), so there is no need to estimate if the $\hat{\omega}$ equals $\omega$ or not. However, the higher accuracy of frequency estimation, the better the phase difference measurement.

\section{3) The new DTFT algorithm based on 4-order RSCW}

The time domain and frequency domain of 4-order RSCW expressions are illustrated in equation 5 and 6 , respectively. According to the derivation process of the new DTFT algorithm based on 2-order RSCW for phase difference measurement, the new DTFT algorithm based on 4-order RSCW for phase difference calculation can be obtained similarly as 


$$
\Delta \theta=\arctan \left[\frac{m_{5}\left(\tan \varphi_{R^{4}, 2 N}-\tan \varphi_{R^{4}, 1 N}\right)}{m_{6}+m_{7}\left(\tan \varphi_{R^{4}, 1 N}+\tan \varphi_{R^{4}, 2 N}\right)+m_{8} \tan \varphi_{R^{4}, 1 N} \tan \varphi_{R^{4}, 2 N}}\right]
$$

Where

$$
\begin{aligned}
& m_{5}=(N \sin \hat{\omega} / 4)^{8}-\sin ^{8} \beta ; \\
& m_{6}=(N \sin \hat{\omega} / 4)^{8}+\sin ^{8} \beta-2 \cdot\left[(N \sin \hat{\omega} \sin \beta / 4)^{4} \cos 4 \beta\right] ; \\
& m_{7}=2 \cdot\left[(N \sin \hat{\omega} \sin \beta / 4)^{4} \sin 4 \beta\right] ; \\
& m_{8}=(N \sin \hat{\omega} / 4)^{8}+\sin ^{8} \beta+2 \cdot\left[(N \sin \hat{\omega} \sin \beta / 4)^{4} \cos 4 \beta\right] ; \\
& \beta=N \hat{\omega} / 4
\end{aligned}
$$

\section{The sliding recursive DTFT algorithm.}

If the signals are time-varying, the algorithm of DTFT cannot be carried out directly. As the width value of window $N$ is very small and the frequency varies a bit in the window which can be approximately reckoned, the frequency does not change in the window, and then, the phase difference of each sampled datum can be worked out based on the sliding intercepting thought.

For signal $x(n)$, assume that $N$ data have been sampled at the time of $m$, i.e. $x(m), x(m+1) \ldots x(m+N-1)$, The DTFT of the sampling sequence at $\hat{\omega}_{m}$ can be expressed as

$$
\begin{aligned}
& X_{m, N}\left(\hat{\omega}_{m}\right)=\sum_{n=0}^{N-1} x(m+n) \cdot e^{-j \hat{\omega}_{n} n} \\
& \quad=x(m)+x(m+1) * e^{-j \hat{\omega}_{m}}+x(m+2) * e^{-2 j \hat{\omega}_{m}}+\cdots+x(m+N-1) * e^{-j \hat{\omega}_{m}(N-1)}
\end{aligned}
$$

At the time of $m+1$, a new datum $x(m+N)$ is sampled and added to the data, while the $x(m)$ is eliminated from the data, the DTFT of the sequence with $N$ data at $\hat{\omega}_{m+1}$ can be expressed as

$$
\begin{aligned}
& X_{m+1, N}\left(\hat{\omega}_{m+1}\right)=\sum_{n=0}^{N-1} x(m+1+n) \cdot e^{-j \hat{\omega}_{n+1} n} \\
& \quad=x(m+1)+x(m+2) * e^{-j \hat{\omega}_{m+1}}+\cdot \cdot+x(m+N-1) * e^{-j \hat{\omega}_{n+1}(N-2)}+x(m+N) * e^{-j \hat{\omega}_{m+1}(N-1)}
\end{aligned}
$$

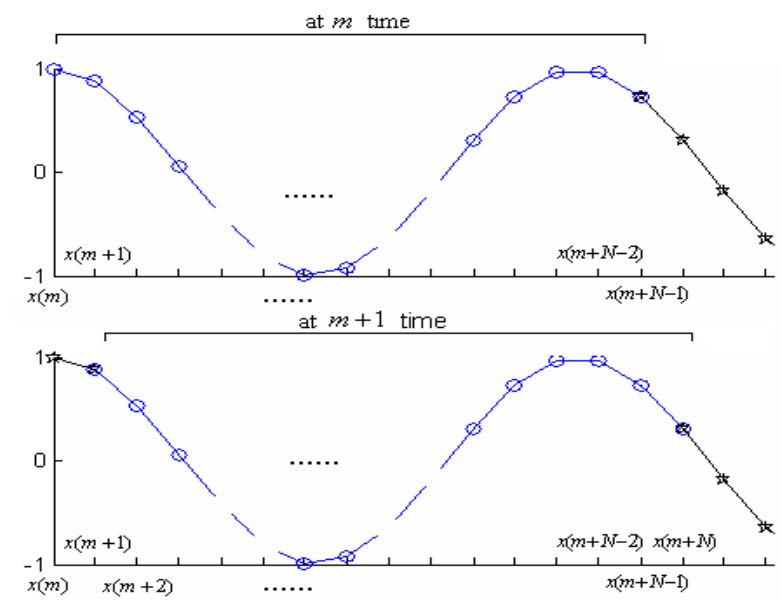

Fig.1. The sliding time window for $N$ points.
Comparing (18) and (19), we observe that $\hat{\omega}_{m}$ and $\hat{\omega}_{m+1}$ delegate the estimated frequency of two adjacent window sampling points, respectively. It is obvious from Fig.1. that it is inaccurate to use $\hat{\omega}_{m+1}$ to calculate the $X_{m+1, N}\left(\hat{\omega}_{m+1}\right)$, as the $N-1$ points are the same in the two adjacent windows. So, (19) can be revised as follows

$$
\begin{aligned}
X_{m+1, N}\left(\hat{\omega}_{m+1}\right) & =x(m+1)+x(m+2) * e^{-j \hat{\omega}_{n+1}}+\cdot \cdot+x(m+N-1) * e^{-j \hat{\omega}_{m+1}(N-2)}+x(m+N) * e^{-j \hat{\omega}_{m+1}(N-1)} \\
& =\left[\sum_{n=0}^{N-1} x(m+n) \cdot e^{-j \hat{\omega}_{m+1} n}-x(m)\right]^{*} e^{j \hat{\omega}_{n+1}}+x(m+N) * e^{-j \hat{\omega}_{n+1}(N-1)} \\
& \approx\left[\sum_{n=0}^{N-1} x(m+n) \cdot e^{-j \hat{\omega}_{n} n}-x(m)\right] * e^{j \hat{\omega}_{m+1}}+x(m+N) * e^{-j \hat{\omega}_{n+1}(N-1)} \\
& =\left[X_{m, N}\left(\hat{\omega}_{m}\right)-x(m)+x(m+N) * e^{-j \hat{\omega}_{n+1} N}\right] * e^{j \hat{\omega}_{n+1}}
\end{aligned}
$$

Equation 20 is the sliding recursive DTFT algorithm proposed in this paper. It is obvious that there is a recursive relationship exit among the new sampling sequence with the old one. Besides, only 2 complex additions and 2 complex multiplications are required to calculate the new DTFT, and the routine DTFT calculation is just needed at the first rectangular window in the whole process, which discards the redundancy calculations. Note that the equation 20 is quite suitable for the signal when the frequency is steady. For time-varying signal, if all $\hat{\omega}_{m+1}$ which exist in the first underline part in (20) are displaced by $\hat{\omega}_{m}$, the dynamic characteristic will be weakened, especially if the frequency varies in a big range.

As for the typical DTFT-based algorithm, the routine DTFT calculation is needed in every window without exception. When a new datum is obtained, $N$ complex multiplications and $N-1$ complex additions are required to calculate the new DTFT. Besides, according to Fig.1., the conventional DTFT-based algorithm has $N$-1 redundancy calculations.

\section{The steps of the proposed algorithm.}

To sum up, the steps of the proposed algorithms can be illuminated as follows

(1) Estimate the signal frequency by adaptive notch filter or discrete spectrum correction, denoted by $\hat{\omega}_{i}$;

(2) Calculate the DTFTs by (9) at the first rectangular window at $\hat{\omega}_{1}$;

(3) Calculate $\tan \varphi_{1}$ and $\tan \varphi_{2}$ by convolving the 2-order RSCW or 4-order RSCW with DTFT in the frequency domain;

(4) Calculate $m_{1}$ to $m_{4}$ or $m_{5}$ to $m_{8}$ by $\hat{\omega}$ and $N$, and then substitute into (16) or (17) for $\tan \varphi_{1}$ and $\tan \varphi_{2}$ to calculate the phase difference;

(5) Make use of the sliding recursive DTFT algorithm in (20), calculate the DTFT at every frequency point $\hat{\omega}_{i}$;

(6) Repeat step (3) and step (4), the phase difference can be obtained at any time. 


\section{SIMULATION AND EXPERIMENTAL RESULTS}

In order to validate the effectiveness of the proposed method, computer simulations have been carried out firstly. To draw a comparison, the phase difference estimates are given at different conditions, such as under noisy or noiseless circumstance, under different sampling data circumstance, and so on. Then, experimental data under different flow rates is gathered through the Coriolis mass flowmeter platform designed by our research team, which is used for experiments. In simulations, the phase difference equals $1.8^{\circ}$, the sampling frequency equals $1000 \mathrm{~Hz}$, the number of sampled points equals 1024, and the frequency resolution equals $0.9766 \mathrm{~Hz}$.

\section{A. Simulation results.}

Under noiseless circumstances, the relative errors of phase difference which are computed by comparing the estimated values of phase difference versus the theoretic values are shown in Fig.2. and Fig.3., respectively. The signal frequency varies from $1 \mathrm{~Hz}$ to $10 \mathrm{~Hz}$ in Fig.2., and varies from $490 \mathrm{~Hz}$ to $499 \mathrm{~Hz}$ in Fig.3., with the step length of $0.1 \mathrm{~Hz}$.

As shown in Fig.2. and Fig.3., the DTFT-based algorithm causes significant errors, while the accuracy of the proposed algorithms is quite low, and they are always superior to the DTFT-based algorithm in the absence of noise. This is because the side lobe of negative frequency components is considered in the proposed algorithms. Besides, the accuracy of the proposed algorithm with the 4-order RSCW is superior to the one with 2-order RSCW, for the 4-order RSCW has a lower attenuation and better inhibition of spectral leakage than 2-order RSCW.

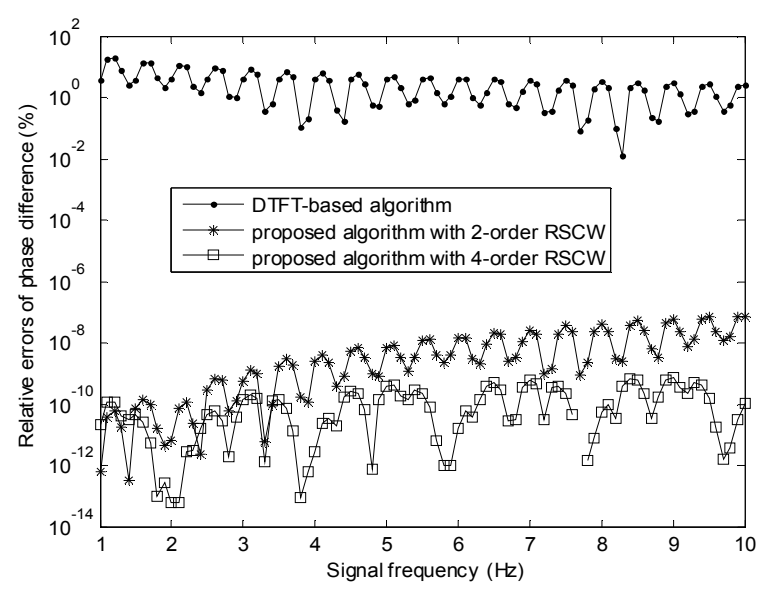

Fig.2. Relative errors of phase difference in the absence of noise when the signal frequency is quite low.

For real-valued signals in the presence of white Gaussian noise, extensive computer simulations have been conducted to evaluate the performance of the proposed algorithms. The white Gaussian noises imposed on two sinusoids are not correlative. Comparisons are also made by the DTFT-based algorithm with the proposed algorithms. All simulation results provided are the average of 100 independent runs.

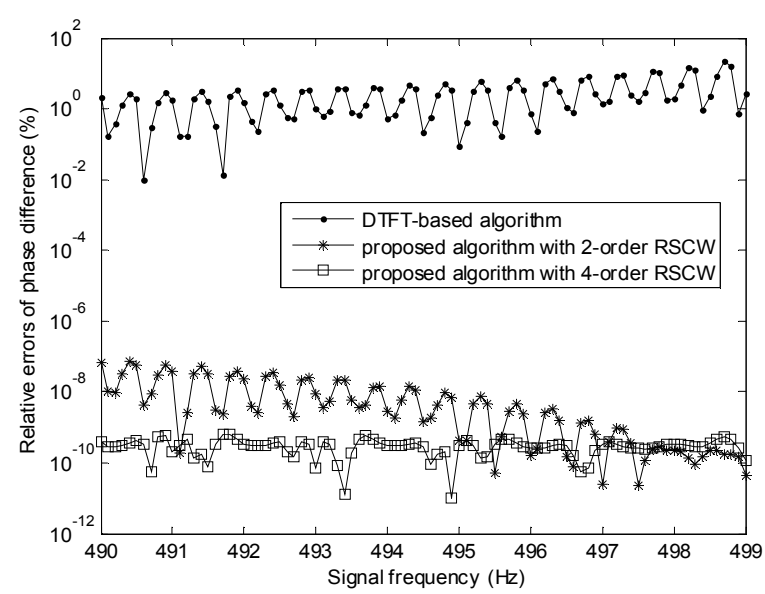

Fig.3. Relative errors of phase difference in the absence of noise when the signal frequency is close to Nyquist frequency.

Fig.4. and Fig.5. show the MSE of the proposed algorithms and DTFT-based algorithm versus the signal frequency at $\mathrm{SNR}=20 \mathrm{~dB}$, respectively. It is observed from Fig.4. and Fig.5. that the proposed algorithms are almost unbiased, while the DTFT-based algorithm results in a significant bias when the signal frequency is quite low or close to the Nyquist frequency. The bias of DTFT-based algorithm varies in the manner of decaying oscillation, because the side lobe of negative frequency components in the spectrum equals to zero, when signal frequency equals to a multiple of half a frequency resolution. In other words, the contribution of negative frequency can be ignored at this moment. What is more, the MSE of the DTFT-based algorithm approach those of the proposed algorithms asymptotically when the signal frequency moves away from zero and Nyquist frequency.

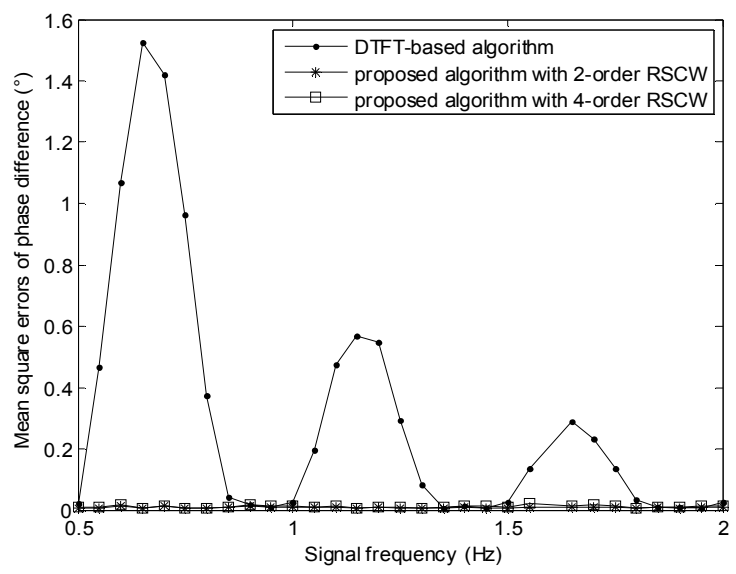

Fig.4. MSE of phase difference under $\mathrm{SNR}=20 \mathrm{~dB}$ when the signal frequency is quite low.

Fig.6. shows the MSE of the proposed algorithms, and DTFT-based algorithm versus the different sampling points varies from 100 to 1000 when signal frequency equals $2.5 \mathrm{~Hz}$. It is seen that the DTFT-based algorithm approaches those of the proposed algorithms asymptotically when the signal frequency moves away from zero and Nyquist frequency, besides, the biases of DTFT-based algorithm vary in the manner of decaying oscillation. Both of these reasons are illuminated as shown in Fig.4. and Fig.5. 


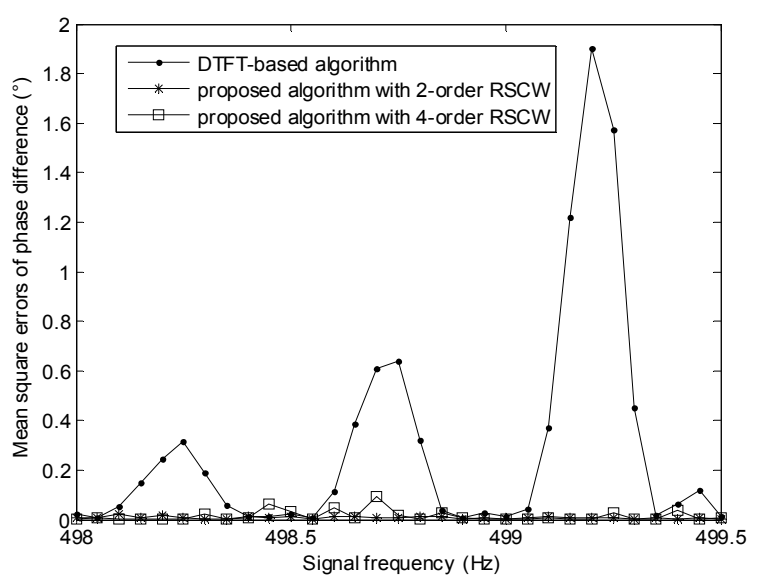

Fig.5. MSE of phase difference under $\mathrm{SNR}=20 \mathrm{~dB}$ when the signal frequency is close to Nyquist frequency.

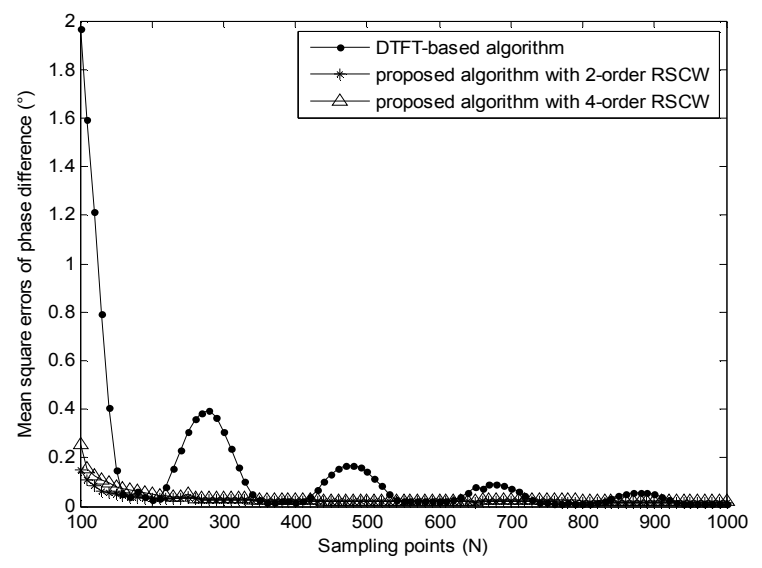

Fig.6. MSE of phase difference under different sampling points.

Fig.7. shows the comparison of phase difference versus the sampling points estimated by the DTFT-based algorithm and the proposed algorithm with 4-order RSCW. We just present the proposed algorithm with 4-order RSCW, for the accuracy of the proposed algorithm with 2-order RSCW is quite close to the proposed algorithm with 4-order RSCW, and the accuracy of the proposed algorithm with the 4-order $\mathrm{RSCW}$ is superior to the one with 2-order RSCW. As is shown in Fig.7., the proposed algorithm can track the phase difference continuously, while the DTFT-based algorithm causes significant errors with the increase of sampling points, because the proposed algorithm adopts the sliding recursive idea, which can eliminate the redundancy calculation of DTFT. The proposed algorithm is of less computer load and has better dynamic performance.

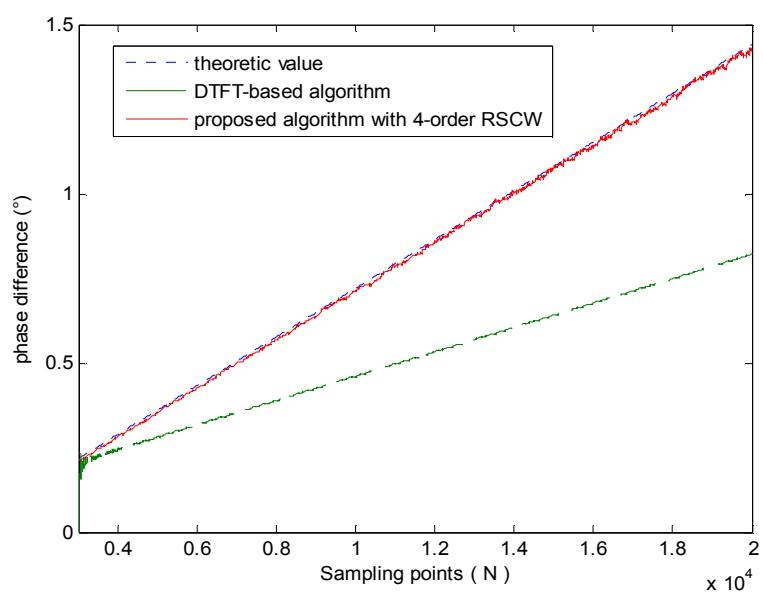

Fig.7. The comparison curve of phase difference estimated by two algorithms.

\section{B.. Experimental results.}

In order to discuss the effectiveness in practice, making use of the CMF plant which our group developed to acquire large numbers of data, then the DTFT-based algorithm and the proposed algorithm with 4-order RSCW are evaluated. For $\mathrm{CMF}$, the mass flow rate is calculated by measuring the phase difference or time interval between two signals detected by electromagnetic sensors. We selected the RHEONIK CMF with a RHE08 transmitter in the experiments, the range of the mass flow rate varies from $0.40 \mathrm{~kg} / \mathrm{min}$ to $16.84 \mathrm{~kg} / \mathrm{min}$, and the signal frequency of CMF approximates $146 \mathrm{~Hz}$. At each steady flow rate, 20000 sampling points of data are sampled each time for each sensor.

Table 1. shows the estimated time delays and relative errors under different flow rates. As shown in Table 1., the results of the proposed algorithm are much closer to the theoretic values of the time delays by comparison, whereas the DTFT-based algorithm causes remarkable deviations, which also validate the effectiveness of the proposed algorithm.

Table 1. The estimated time delays and relative errors under different flow rates.

\begin{tabular}{|c|c|c|c|c|c|}
\hline \multirow{2}{*}{$\begin{array}{l}\text { Mass flow } \\
\text { rate } \\
/(\mathrm{kg} / \mathrm{min})\end{array}$} & \multirow{2}{*}{$\begin{array}{l}\text { The theoretic } \\
\text { time delay } \\
\text { values/ } \mu \mathrm{s}\end{array}$} & \multicolumn{2}{|c|}{ The mean of time delay values/ $\mu \mathrm{s}$} & \multicolumn{2}{|c|}{ The relative error of time delay values $/ \%$} \\
\hline & & $\begin{array}{c}\text { DTFT-based } \\
\text { algorithm }\end{array}$ & $\begin{array}{l}\text { The proposed algorithm } \\
\text { with 4-order RSCW }\end{array}$ & $\begin{array}{l}\text { DTFT-based } \\
\text { algorithm }\end{array}$ & $\begin{array}{l}\text { The proposed algorithm } \\
\text { with 4-order RSCW }\end{array}$ \\
\hline 0.40 & 2.9146 & 2.9287 & 2.9185 & 0.4838 & 0.1338 \\
\hline 1.12 & 8.0847 & 8.1243 & 8.0956 & 0.4898 & 0.1348 \\
\hline 2.28 & 16.4143 & 16.4961 & 16.4365 & 0.4983 & 0.1352 \\
\hline 4.36 & 31.3502 & 31.5106 & 31.3928 & 0.5116 & 0.1359 \\
\hline 8.50 & 61.0783 & 61.4098 & 61.1627 & 0.5427 & 0.1382 \\
\hline 16.84 & 120.9653 & 121.6971 & 121.1341 & 0.6050 & 0.1395 \\
\hline
\end{tabular}




\section{CONCLUSION}

The DTFT-based algorithm for phase difference measurement is biased when the frequency is quite low or close to the Nyquist frequency. In order to improve the accuracy of the DTFT-based algorithm, two new sliding DTFT algorithms for phase difference measurement based on a new kind of windows have been developed, and the phase difference calculation formulas with 2-order RSCW and 4-order RSCW are presented, respectively. The proposed algorithms considering the negative frequency contribution, adopting the RSCW and the sliding recursive idea, can remove the bias of the DTFT-based algorithm and attain optimum performance all the time, especially for the proposed algorithm with 4-order RSCW. Simulations and experimental results validate the effectiveness of the proposed algorithms.

For future research, we will focus on extending and generalizing this type of algorithms to a more general system identification scheme, and further research is under discussion.

\section{ACKNOWLEDGMENT}

This work was supported by the National Natural Science Foundation of China $(61271449,61302175)$ and the Natural Science Foundation Project of Chongqing (2012jjA40006, 2013jcyjA40030).

\section{REFERENCES}

[1] Etter, D., Strarns, S. (1981). Adaptive estimation of time delays in sampled data systems. IEEE Transactions on Acoustics, Speech and Signal Processing, 29 (3), 582-587.

[2] Maskell, D.L., Woods, G.S. (1999). The estimation of subsample time delay of arrival in the discrete-time measurement of phase delay. IEEE Transactions on Instrumentation and Measurement, 48 (6), 1227-1231.

[3] Tu, Y.Q., Zhang, H.T. (2008). Method for CMF signal processing based on the recursive DTFT algorithm with negative frequency contribution. IEEE Transactions on Instrumentation and Measurement, 57 (11), 2647-2653.
[4] Ramos, P.M., Cruz Serra, A. (2008). A new sine-fitting algorithm for accurate amplitude and phase measurements in two channel acquisition systems. Measurement, 41 (2), 135-143.

[5] Vucijak, N.M., Saranovac, L.V. (2010). A simple algorithm for the estimation of phase difference between two sinusoidal voltages. IEEE Transactions on Instrumentation and Measurement, 59 (12), 3152-3158.

[6] Shen, T.A., Tu, Y.Q., Zhang, H.T. (2014). A novel time varying signal processing method for Coriolis mass flowmeter. Review of Scientific Instruments, 85 (6), 065116.

[7] Knapp, C.H., Carter, G.C. (1976). The generalized correlation method foe estimation of time delay. IEEE Transactions on Acoustics, Speech and Signal Processing, 24 (4), 320-327.

[8] Carter, G.C. (1993). Coherence and Time Delay Estimation: An Applied Tutorial for Research, Development, Test, and Evaluation Engineers. IEEE Press.

[9] Maskell, D.L., Woods, G.S. (2005). Adaptive subsample delay estimation using a modified quadrature phase detector. IEEE Transactions on Circuits and Systems, 52 (2), 669-674.

[10] So, H.C. (2001). Time-delay estimation for sinusoidal signals. IEE Proceedings - Radar, Sonar and Navigation, 148 (6), 318-324.

[11] Maskell, D.L., Woods, G.S. (2002). The discrete-time quadrature subsample estimation of delay. IEEE Transactions on Instrumentation and Measurement, 51 (1), 133-137.

[12] So, H.C. (2005). A comparative study of two discrete-time phase delay estimators. IEEE Transactions on Instrumentation and Measurement, 54 (6), 2501-2504.

[13] Huang, C., Jiang, Y.Q. (2005). Improved window and interpolation algorithm for analysis of power system harmonics. Proceedings of the CSEE, 25 (15), 26-32.

[14] Oppenheim, A.V., Schafer, R.W. (1989). Discrete-Time Signal Processing. Prentice-Hall.

Received May 19, 2014. Accepted October 30, 2014 